\title{
Critères intervenant dans le choix de l'expert judicaire, psychiatre ou psychologue, en France
}

\author{
PALARIC R, MOULIN V.
}

Publié, aux Annales Médico-Psychologiques, Vol 172/7, 489-494, 2014.

\section{Introduction}

Personnages de la scène judiciaire devenus incontournables, les experts occupent aujourd'hui une place prépondérante dans de nombreux procès. En France, le recours à l'expert psychiatre et psychologue est particulièrement fréquent, voire dans certains cas automatique. Dans cet article, nous interrogeons les raisons du choix d'un expert, psychiatre ou psychologue, par le magistrat, à la lumière des relations juge-expert.

Dans l'histoire française, le recours à la pratique expertale s'est progressivement développé par une sollicitation de plus en plus importante des experts (textes légaux). Ces derniers sont sollicités directement par les magistrats afin de procéder à une expertise psychiatrique ou psychologique. L'article 157 du Code de Procédure Pénale français prévoit les modalités de désignation d'un expert par le magistrat : "Les experts sont choisis parmi les personnes physiques ou morales qui figurent sur la liste nationale dressée par la Cour de cassation ou sur une des listes dressées par les cours d'appel dans les conditions prévues par la loi n 71-498 du 29 juin 1971 relative aux experts judiciaires. A titre exceptionnel, les juridictions peuvent, par décision motivée, choisir des experts ne figurant sur aucune de ces listes". Le choix de l'expert n'est contraint que par la présence de celui-ci sur une liste tout en laissant au magistrat la possibilité de s'en extraire, à condition de pouvoir motiver cette décision. Les experts peuvent être sollicités à différents moments du parcours pénal : en phase d'enquête, avant le jugement (phase d'instruction), en cours d'exécution des peines/mesures et également au terme de celles-ci. Initialement, l'expert était sollicité lorsque le magistrat se posait une question relevant de compétences spécifiques, alors qu'aujourd'hui le recours à l'expert peut être perçu comme une obligation légale, pas toujours nécessaire.

La recherche sur laquelle se base cet article visait à interroger les relations « juge-expert » et à proposer une lecture de la dynamique expertale, tant du point de vue juridique que clinique. Il s'agissait principalement d'interroger les enjeux explicites et implicites de ces relations et les processus qu'elles suscitent chez chacun des protagonistes, en considérant que la dynamique expertale peut susciter un processus de "co-construction" expertale, connue ou méconnue par ses acteurs. La co-construction est généralement perçue négativement, dans ses «aveuglements », comme une « cooptation » préjudiciable aux intérêts de la justice. La notion « de couple» [8], de «binômes juge-expert » est évoquée pour relever les jeux d'influence et la suggestion de l'un sur l'autre. Certains évoquent le pouvoir des experts, d'autres ceux des juges. Ce jeu d'influences serait susceptible de porter atteinte au «monopole du droit dans la définition du juste » [6]. Les relations juge-expert peuvent aussi être pensées positivement, par l'intermédiaire des relations de confiance professionnelle et dans une dialectique intégrative, inscrivant la pluridisciplinarité et les échanges dans une dimension constructive, visant la compréhension et l'adaptation de la réponse pénale 
Dans cet article, nous nous centrerons sur la présentation des éléments qui sous-tendent le choix d'un expert par les magistrats. Partant de la présentation des deux types de systèmes de justice inquisitoire et accusatoire, nous restituerons la place du juge et de l'expert dans ces deux sytèmes et le statut de ce dernier, entre expert judiciaire et expert witness. L'exemple de pratiques étrangères sur la désignation de l'expert nous permettra de spécifier les modes de désignation de l'expert français. A partir des résultats empiriques d'une recherche, nous présenterons de manière descriptive des critères de choix d'un expert énoncés par les magistrats, ainsi que les pôles d'influence qui semblent sous-tendre ce choix.

\section{1- Mode de désignation de l'expert judiciaire en France et quelques exemples à l'étranger}

\section{1-1. $\quad$ Statut du discours expertal : les systèmes Inquisitoire et Accusatoire}

Deux grands systèmes judiciaires cohabitent à l'échelle internationale, le système accusatoire et le système inquisitoire.

Pour le premier, principalement situé dans les pays issus de la common law, il s'agit pour les parties d'apporter des éléments utiles à la cause défendue. Dans ce système, les parties sont les deux seuls maîtres du procès. On dit traditionnellement que le procès "est la chose des parties", celles-ci désignant le mis en examen et le Ministère Public. Aucune place n'est faite à la victime. Le juge se positionne alors en "arbitre", mesurant les arguments de chacun. Il n'a qu'un rôle passif, se cantonnant à veiller au respect des règles de procédures, puisque ce sont les parties qui ont l'initiative de la preuve. Le juge assure le bon déroulement de la mise en concurrence loyale des (deux) récits.

Le système inquisitoire place le juge au centre de l'enquête puisqu'il représente la société. Il a un rôle actif, le terme Inquisition désignant étymologiquement Enquête. C'est au "juge enquêteur à qui il revient d'établir la version officielle" [11]. A la différence du modèle accusatoire, la justice dépasse l'intérêt des parties et s'intéresse aussi à la société, formant ainsi une troisième entité durant le procès ; le ministère public pouvant par exemple faire appel d'une décision. La victime est également partie intégrante du procès par le dépôt de plainte avec ou sans constitution de partie civile ou citation directe [14].

Cette différenciation des systèmes judiciaires implique une distinction du statut de l'expert et des effets de sa parole. Tout d'abord, du point de vue de la désignation comme "expert".

$\mathrm{Si}$, en France, l'inscription sur une liste officielle implique des modes de contrôle de l'expérience et des compétences du professionnel, le revers de cette pratique serait de voir un expert nommé ad vitam aternam, s'il peut justifier de formations dans le champ considéré afin de demander sa réinscription. Depuis 2004, des réformes introduisent des évaluations, avec possibilités de sanction, tout au long de la carrière de l'expert. De même, une démarche de réinscription sur la liste, tous les cinq ans, a été instaurée ${ }^{1}$.

A l'inverse, dans le système accusatoire, "il est considéré que la justice n’a pas les compétences pour juger des capacités d'un expert" [10]. L'expert est donc un professionnel pouvant intervenir "sur tout sujet

\footnotetext{
${ }^{1}$ Décret du 23 décembre 2004, art.10 : "Les demandes de réinscription pour une durée de cinq ans sont envoyées avant le 1er mars de chaque année au procureur de la République"
} 
pertinent sur lequel il est qualifié pour apporter des preuves" [13]. (Royaume-Uni, article 3-1 de la loi sur les preuves en matière civile). Ainsi, si aux Etats-Unis, devant l'inexistence des listes d'experts, les parties peuvent proposer l'expert de leur choix, il appartient tout de même au juge de tenir son rôle de Gatekeeper, chargé de vérifier les compétences dudit expert [3] (Cf. infra exemples à l'étranger). Le contrat ne lie plus alors l'expert au juge, mais directement aux parties qui l'ont sollicité, puisqu'il leur appartient de rédiger les missions expertales.

\section{1-2. Expert judiciaire et expert witness}

En répartissant les pouvoirs d'enquête sur le juge ou les parties, les systèmes judiciaires que nous opposons offrent aux experts des statuts différents.

En France, l'expertise au pénal constitue une pratique encadrée sur le plan légal. L'expert peut ou doit être sollicité pour certaines catégories infractionnelles, en vue d'apporter des éléments techniques sur des points particuliers. Les experts, psychiatres ou psychologues, sont considérés comme des «techniciens» (Code de Procédure Pénale) qui acceptent d'être au service de la justice. Ils ont à répondre aux missions qui leur sont confiées par les magistrats, en fonction de leurs compétences et connaissances dans le champ considéré (Loi du 11 février 2004 sur le statut des experts). La loi de 1971 et le décret de 1974 ont permis l'instauration d'un «statut d'expert» par la mise en place d'un titre légal d'expert judiciaire et l'instauration d'une liste locale des experts (par Cour d'Appel) et ainsi qu'une liste nationale. Les psychiatres et psychologues experts sont des professionnels inscrits sur les listes. Seule l'inscription sur les listes confère au praticien la qualité et le titre d'expert. L'inscription sur les listes suppose de remplir différents critères, dont le premier correspond aux «bonnes mœurs» (en particulier l'absence de condamnation pénale); aux compétences des experts (formation et activités antérieures et en cours ; à leur indépendance vis-àvis des missions d'expertise ; également des critères concernant l'âge et le lieu de résidence. La décision d'inscription est prise par les juridictions, après que le praticien ait présenté un dossier qui atteste de ses compétences. Les listes d'experts sont revues tous les quatre ans, mais l'expert doit attester annuellement de son activité et de l'actualisation de ses connaissances (formations) dans le champ [12].

En France, seul le juge peut désigner un expert, s'il l'estime opportun ou si l'infraction le nécessite. Ce dernier point dépasse le stade de l'instruction et place le juge dans une position particulière car, s'il ne souhaite pas solliciter d'expert, il doit motiver sa non-demande ; conduisant Faucher à dire que "l'on est passé de la faculté d'ordonner une expertise à l'obligation d'expertise. Ainsi, l'on ne demande pas au juge de l'application des peines de discerner les cas où cette expertise peut être utile" [9]. Depuis 2007, si les avocats 
peuvent conseiller la nomination d'un expert particulier ${ }^{2}$, cette décision reste du pouvoir du juge. L'expert nommé fournira alors ses conclusions au juge, seul professionnel habilité à recueillir les informations utiles au déroulement du procès et à la manifestation de la vérité.

Dans les pays de la Common law, les experts sont missionnés par les parties elles-mêmes et payés par elles, ce qui fait dire à certains auteurs que "l'expert est à la solde de la partie qui le fait entendre, donc partial par définition" [2]. Les informations fournies constituent alors une preuve, dont la valeur est jugée supérieure à celle d'un témoin non compétent ; l'expert étant alors qualifié d' "expert witness". En raison de la possible multiplication des points de vue, il peut incomber au juge de statuer sur des controverses scientifiques, qu'il n'est pourtant pas en mesure d'arbitrer. Selon les pays ou états, des règles spécifiques sont mises en place.

\section{1-3. Exemple de pratiques étrangères}

- USA : Jusqu'en 1923, le système judiciaire américain considérait qu'un professionnel pouvait devenir expert judiciaire s'il démontrait que son activité lui permettait de gagner sa vie. La réussite professionnelle attestait ainsi des compétences [7]. A partir de 1923, l'arrêt FRYE encadre la fonction de l'expert car celui-ci doit présenter des informations exprimant la position de l'ensemble de la communauté scientifique (critère du general acceptance standard). Une première difficulté apparaît : il est en effet difficile pour le juge de vérifier l'exactitude des informations apportés par l'expert sans lui-même devenir un professionnel du domaine concerné. A une période de forte évolution des savoirs, cette fonction apparaît complexe. Ainsi, en 1985, l'arrêt DAUBERT spécifie les exigences vis-à-vis des experts. Quatre axes précisent les critères de scientificité requis pour que le « témoignage » de l'expert soit recevable :

-les théories et méthodes utilisées doivent pouvoir être testées et réfutées ;

-les théories avancées doivent avoir été soumises au regard des pairs et publiées ;

-il doit exister un pourcentage d'erreur connu ;

-le critère de general acceptance standard (norme d'acceptation) doit être connu.

Le juge (à travers son rôle de Gatekeeper) doit vérifier la fiabilité du témoignage de l'expert. Dans cet objectif, des rencontres entre experts, juges et parties (déposition) ont lieu, avant le procès, durant lesquelles les experts démontrent leur méthodologie. Parallèlement à la constitution de ces deux arrêts, l'utilisation de concepts scientifiques est requise à des fins d'argumentation juridique. Le concept de Junk science (Junk= rebus) définit les informations présentées comme scientifiques mais qui ne présentent aucun fondement scientifique. Le concept de Sound science concerne les applications scientifiques de données orientées vers le point de vue de l'une des parties. L'émergence de tels concepts montre l'intérêt, aux USA, de garantir un cadre de recours aux experts.

Les experts sont soumis à des règles extrêmement exigeantes, celui "qui rend un témoignage insoutenable sur le plan scientifique risque de se discréditer irrémédiablement de la communauté scientifique"

\footnotetext{
${ }^{2}$ Article 161-1 CPP : "Copie de la décision ordonnant une expertise est adressée sans délai au procureur de la République et aux avocats des parties, qui disposent d'un délai de dix jours pour demander au juge d'instruction, selon les modalités prévues par l'avantdernier alinéa de l'article 81, de modifier ou de compléter les questions posées à l'expert ou d'adjoindre à l'expert ou aux experts déjà désignés un expert de leur choix figurant sur une des listes mentionnées à l'article 157".
} 
[2]. De même, il existe des sanctions possibles des Ordres professionnels si les parties, qui attendent de l'expert "un service", ne sont pas satisfaites, elles n'hésitent pas à saisir le Syndic de l’Ordre. Cela caractérise bien la relation contractuelle liant l'expert au client (terme communément retenu par les experts pour qualifier la partie).

- Espagne : l'Espagne propose, en termes d'expertises, un régime mixte original. Il est possible, dans un même dossier de voir des experts missionnés par le juge, comme par les parties. Plus précisément, le juge a la liberté de saisir un expert uniquement dans le cas où une partie le souhaite [5]. Le discours expertal prend donc un statut particulier, puisqu'il vient concerner à la fois l'accusation et la défense, mais aussi la position judiciaire.

- Allemagne : il appartient au juge de décider de la nécessité du recours à l'expertise, "selon sa libre conviction". Le tribunal désigne alors un expert sur une liste, tant qu'il en existe pour la discipline concernée. Un point particulier apparait lorsque les parties se sont préalablement accordées sur la nomination d'un expert, auquel cas, le juge ne peut y déroger. L'article 404 du Code de Procédure Civile allemand prévoit aussi que les missions demandées à l'expert soient rédigées par le juge, de même que la méthode qui devra être utilisée. Ce dernier point ne semble pas être pris en compte, le juge préférant laisser à l'expert la liberté du choix de la méthode [1].

- L'expert conciliateur : dans certains états, l'expert peut venir jouer le rôle de médiateur entre les parties. Rare en ce qui concerne les affaires criminelles violentes, la conciliation préalable au procès peut se présenter en matière d'accidents médicaux [10]. Dans le but d'économiser des frais de justice, la conciliation vise à trouver rapidement un terrain d'entente entre les parties. Si tel est le cas, une majoration du salaire de l'expert peut être prévue, comme en Israël, où elle peut monter à $15 \%$. Ces mêmes $15 \%$ peuvent être ajoutés au montant de l'indemnisation du plaignant, si la conciliation a lieu avant le procès.

$\mathrm{Ce}$ rapide détour par quelques pratiques étrangères montre que comparativement, le système français a mis en place un dispositif qui garantit au magistrat la maîtrise du dispositif décisionnel dans le choix de l'expert [4]. Seule personne habilitée à faire appel à un expert, et à le nommer personnellement, le juge a donc la responsabilité, par comparaison avec le modèle américain, de choisir l'expert qu'il juge compétent et susceptible de lui apporter des éléments de réponse aux questions posées. Cette responsabilité est cependant relative, car l'expert est inscrit sur une liste qui le désigne comme compétent, avec toutefois la possibilité de recourir à un expert non inscrit si la mission nécessite des compétences ou connaissances très particulières. Rappelons cependant qu'en France, pour les experts psychiatres, un département sur deux connaît une pénurie d'experts, telle que le choix de ceux qui restent disponibles pourrait s'imposer au juge.

\section{Etude empirique : le choix de l'expert}

\subsection{Méthodologie}

Dans le cadre d'une étude plus globale sur la "co-construction expertale" [15], 20 entretiens semidirectifs de recherche ont été menés auprès de professionnels de la justice (Présidents de Cour d'Assises, Juges d'Instruction, Juge de l'Application des Peines et Avocats) afin de répondre à différentes questions, 
notamment celles relatives aux critères de choix de l'expert par le magistrat. A cela s'ajoute trois focus groups de recherche avec des magistrats, au cours desquels ce point, parmi d'autres, a été abordé.

L'entretien semi-directif de recherche a été construit dans le cadre de cette étude, afin de répondre à ses objectifs. Les entretiens ont fait l'objet d'une analyse terminologique et thématique afin de dégager les principales réponses énoncées dans le choix de l'expert. En raison de l'effet de saturation des données ${ }^{3}$ recueillies par l'intermédiaire des entretiens, nous avons approfondi et mis au travail les premiers résultats obtenus, dans le cadre de séminaires organisés sous la forme de focus groups de recherche. Ils consistent dans l'approfondissement d'une problématique déterminée dans le cadre d'un groupe de discussion. Un ou deux modérateurs sont présents dans la séance et guident les interactions sur les thèmes qui sont importants à aborder pour la problématique investiguée. Il s'agit d'une approche qualitative qui permet au chercheur de susciter une discussion ouverte entre les participants sur le sujet d'intérêt pour l'étude et de repérer les enjeux, difficultés qui sous-tendent les thèmes abordés. Les focus groups ont fait l'objet du même type d'analyse que précédemment, associé à une analyse de la dynamique de groupe dans les réponses présentées.

Il s'agit principalement d'une étude qualitative qui a une valeur illustrative car le nombre d'entretiens et de focus groups ne permettent pas la généralisation des résultats obtenus.

\section{2-2. Principaux résultats descriptifs}

\section{2-2.1. Le critère de célérité}

Le premier argument donné par l'ensemble des magistrats et avocats, dans le choix de l'expert, est le critère de célérité. La disponibilité et la rapidité des réponses sont les raisons les plus fréquemment énoncées. Cette réponse, qui vise avant tout à dénoncer le manque d'experts, psychiatres ou psychologues, peut s'analyser comme une priorité donnée à la tâche. Le magistrat cherche à rester dans le cadre de la procédure sur le plan des délais, ou encore à accélérer le processus judiciaire par le choix d'un expert capable de répondre dans les délais impartis.

\section{2-2.2 Le critère de l'habitude}

Le critère de l'habitude est cité comme faisant parti d'un processus de choix de l'expert non-réfléchi, au sens où il ne souffre pas d'une réelle sélection. Dans ce cadre, distinction est faite entre les affaires dites "courantes" et les affaires plus problématiques, aux enjeux ou contextes spécifiques, que le juriste n'a pas pour habitude de traiter. Pour ces premières affaires, l'habitude et la confiance se lient dans une forme d'entente spontanée, dans laquelle le juriste prendrait moins de distance avec les conclusions énoncées. Pour ces affaires classiques, les conclusions de l'expert seraient généralement connues d'avance. En effet, le juriste ayant l'habitude de travailler avec certains experts, il anticiperait les "résultats" d'expertise ; ce qui donne à entendre une forme de stéréotypie dans la production des rapports mais aussi le repérage par les magistrats d'une "typologie des réponses expertales" liées à l'usage. Ainsi le choix peut viser à confirmer une opinion déjà là, consciente ou inconsciente chez le magistrat ; l'expertise permettrait aussi de l'expliciter, de la mettre

\footnotetext{
${ }^{3}$ Nous avons arrêté le nombre d'entretiens à 20 car nous n'obtenions plus d'informations supplémentaires et nous avons approfondi le recueil des données dans le cadre de séminaires de travail.
} 
en mots "à travers les mots de l'expert qui expliquent ce que l'on pensait" (magistrat). Le magistrat s'oriente alors vers celui qui est susceptible de confirmer cette opinion préalable, dans une forme de co-construction qui peut s'ignorer [15].

\section{2-2.3. La relation de confiance}

Si le choix de l'expert s'oriente vers un professionnel en qui le magistrat a confiance, celle-ci se base sur les capacités de l'expert à fournir une expertise dans des délais jugés corrects et de fournir des conclusions "utiles". Le terme "utile" renvoie ici à des informations qui sont qualifiées de "pertinentes" pour la compréhension et l'évolution du dossier. Les informations doivent aussi être accessibles et compréhensibles (par le magistrat et éventuellement les jurés en Cour d'Assises), pour éviter une nouvelle expertise ou un complément. Les termes techniques relevant d'une terminologie psychiatrique ou psychologique doivent être explicités afin d'éviter les confusions et incompréhensions.

\section{2-4. Le domaine de spécialité}

La spécialité n'est pas spontanément argumentée par les magistrats par une spécialisation professionnelle ou universitaire, mais par ce qu'ils pourraient savoir de l'expert. Ce savoir sur l'expert est alimenté par deux types de champs: le premier relève de l'expérience acquise à travers les relations professionnelles entretenues avec l'expert ; faisant de la "spécialité" une conséquence de la co-construction d'une pratique partagée, liée à l'habitude de travailler avec un expert sur des domaines particuliers, mais aussi de la confiance du magistrat envers l'expert sur des questions précises (relatives par exemple à la pédophilie, ou à la cyberpédocriminalité, etc.). Le second renvoie à la "réputation ou renommée", qui impliquent davantage le champ des représentations véhiculées au sein de la profession. D’un point de vue positif, la réputation est principalement reconnue en fonction de la considération d'un expert par d'autres magistrats que celui en position de choix. Cela peut procéder du "bouche à oreille". Dans ce cadre, la confiance est établie entre les deux magistrats. La renommée, aux yeux du magistrat, peut bien sûr être issue de l'expérience et/ou de l'exposition médiatique de l'expert au cours de procès antérieurs.

La notion de spécialité désigne également une distinction entre un travail courant, pour lequel chaque expert peut en remplacer un autre (interchangeabilité des experts), et un travail spécifique pour lequel le recours à l'expertise nécessite une représentation de l'expert aux conclusions inattaquables (renvoyant à la réputation).

Depuis la loi du 5 mars $2007^{4}$, les avocats ont la possibilité de proposer au juge d'instruction de nommer un expert précisément. Si cette loi n'est pas encore appliquée fréquemment, elle ouvre tout de même de nouvelles possibilités dans les rapports entre acteurs judiciaires. En effet, en raison de leur expérience auprès de certains experts, les avocats peuvent envisager à l'avance les conclusions données en fonction du type d'affaire. Ce dernier point peut conduire à recourir plus facilement à certains experts par rapport à d'autres.

\footnotetext{
${ }^{4}$ L'expertise en matière pénale deviendra également plus contradictoire : les parties seront informées de la décision du juge ordonnant une expertise ; elles pourront demander la désignation d'un co-expert de leur choix et faire des observations sur des rapports d'étapes ou sur des pré-rapports. (Loi n 2007-291 du 5 mars 2007 tendant à renforcer l'équilibre de la procédure pénale publiée au Journal Officiel du 6 mars 2007)
} 


\section{2-5. La sollicitation d'une discipline}

Nous intéressant spécifiquement à la psychiatrie et la psychologie, nous distinguons les modes d'appréhension de ces disciplines par les magistrats. Selon les personnes interrogées, le recours à l'expert psychiatre est une exigence légale qui vise à interroger les notions de responsabilité juridique, de dangerosité et d'injonctions de soins. L'expert psychologue apporte une analyse de la personnalité et des faits, qui s'inscrit dans une histoire de vie. Le psychiatre expert serait davantage attendu dans la production d'un "syndrome" en tant qu'ensemble de signes cliniques permettant de rendre compte d'un "état" psychique structuré ou non, auquel s'attacheront les notions de responsabilité et dangerosité. Le psychologue, lui, serait davantage attendu du côté d'une compréhension dynamique et processuelle de la constitution d'une personnalité, de son mode de fonctionnement, de l'analyse de l'acte et du sens de celui-ci dans une histoire.

Les discours recueillis évoquent la notion de valeur ajoutée par laquelle l'expertise doit dépasser la simple réponse aux missions, ou le champ disciplinaire de l'expert, pour enrichir la compréhension du dossier. Une expertise peut être appréciée car elle permet de "fermer des portes", ou fermer des hypothèses dans la démonstration légale; à l'inverse, l'expertise est attendue pour faire advenir de nouvelles questions, de nouvelles hypothèses.

\section{2-6. Une forme de stratégie}

Le choix de l'expert peut aussi relever d'une forme de "stratégie" [6] reposant sur la connaissance de l'expert et de ses habitudes de fonctionnement. L'expert peut ainsi être choisi ou écarté en raison de l'orientation de ses rapports et conclusions, renvoyant comme précédemment au repérage de tendances dans les écrits des experts. Ce choix rationnel s'inscrit dans la volonté de dire le juste, relevant davantage d'un positionnement éthique. La dimension stratégique dans le choix de l'expert peut aussi s'inscrire dans une rationalité procédurale dans laquelle il peut s'agir d'emblée de faire appel à un expert de renom, de manière à ce qu'il soit incontestable ; ou progressivement, en choisissant d'abord un expert peu connu pour aboutir à un choix d'expert reconnu, mais également dans une volonté de faire poids aux assises à travers un expert incontestable, car reconnu comme étant un des spécialistes du champ considéré.

L'expertise peut aussi être envisagée comme une obligation légale, notamment en cas de crime sexuel. Le magistrat peut se contraindre à solliciter un expert alors qu'il n'en perçoit pas l'intérêt, pour des raisons diverses : notamment, par exemple, le poids que peut prendre un rapport d'expertise par rapport à d'autres pièces du dossier. Si le juge d'instruction ne souhaite pas d'expertise, il doit alors motiver l'absence de recours à l'expert.

De manière marginale, un élément participant au choix est énoncé autour de la formation des experts, notamment de leur obédience théorique, susceptible d'influer sur leur réponse. Ce point est évoqué autour de la question de la dangerosité criminologique, notion complexe qui suscite des positionnements divers selon les experts, certains acceptant de se prononcer sur ce point, d'autres non. Ainsi un choix pourrait être réalisé en fonction du positionnement de l'expert, qui sera écarté s'il n'accepte pas de répondre à cette question qui fait partie des obligations légales. 


\section{2-7. Des critères de non-choix}

Les experts peuvent être mis à l'écart, pour trois raisons principales : l'absence de respect des délais dans la réalisation de la mission expertale, renvoyant à un problème d'observation des procédures ; en raison de "l'inconsistance de l'expertise" (magistrat), c'est-à-dire que l'expertise n'apporte pas d'éléments nouveaux ou de nouvelles réflexions; ainsi qu'en raison d'un déficit d'argumentation dans la construction de l'écrit. L'aspect argumentatif apparait particulièrement important tant pour la rigueur de la démarche de l'expert, que pour la compréhension des points développés dans l'expertise (qui ne doivent pas être des postulats mais bien être démontrés), mais aussi afin d'éviter que les avocats s'en saisissent pour remettre en question l'expertise. Ces éléments renvoient à des critères de compétence de l'expert, évalués à travers l'écrit.

La dimension de l'écrit n'est qu'une étape dans le processus de sélection des experts, ces derniers peuvent être écartés en raison de faibles capacités oratoires lors de l'audience en Cour d'Assises. Une "bonne" expertise qui ne peut être portée, argumentée, défendue par l'expert qui l'a rédigée ne répond pas aux attentes des magistrats et de l'institution judiciaire. "Si la loi est écrite, la justice est, comme le rappelle l'étymologie, un dire public". La dimension orale est centrale dans les débats judiciaires. La manière dont l'expert présentera et défendra publiquement son expertise apparaît capitale dans le déroulement de l'audience et, par conséquent, elle peut conditionner le choix de ce dernier. L'expert doit avant tout être pédagogue, éloquent et convaincant dans la présentation de son travail. Dans le choix de l'expert, la capacité d'orateur peut être mise en avant par rapport à la qualité du travail écrit. Son importance serait capitale car elle pourrait faire "basculer un procès" (un magistrat). Les magistrats font cependant une distinction : un "moins bon orateur" n'est pas forcément un mauvais expert ; ce type d'expert se verra davantage confié des expertises en correctionnelle afin d'éviter les difficultés des débats aux assises.

D'autres magistrats siégeant en correctionnelle exprimeront l'importance de la clarté de l'expert dans la rédaction de son rapport et dans l'argumentation démonstrative, lorsqu'il s'agit d'un délit, ce dernier n'étant pas présent pour expliciter les points en suspens en audience de jugement. Les stratégies dans le choix de l'expert peuvent varier suivant le type d'audience. Ces différents points relèvent d'une centration sur la tâche, dans la volonté d'adapter au mieux le choix afin de donner au dossier la meilleure progression.

\section{Conclusion}

A partir des résultats présentés précédemment, nous pouvons distinguer trois champs d'influence dans le choix de l'expert : le champ judiciaire et procédural, en tant qu'il s'agit d'être et de demeurer dans un cadre légal. Il s'agira alors d'efficacité procédurale ("rapidité d'exécution de l'expertise", "disponibilité de l'expert"), voire de stratégie procédurale; le champ de l'axiologie et des représentations qui renvoie au système de valeurs propre à chacun en fonction de son trajet personnel, de la connaissance du dossier, de sa culture, de son système d'appartenance ; le champ de l'expérience dans la construction d'une relation et des "habitudes professionnelles" dans la désignation de l'expert. Ces trois champs se complètent évidemment par la réalité du terrain. 
Il est en effet fréquent de constater que la disponibilité d'un expert dans un temps donné soit le premier critère de choix. De nouvelles organisations peuvent alors se mettre en place, comme la précaution de ne pas surcharger un expert «intéressant» afin de lui attribuer uniquement les dossiers jugés inhabituels. Ainsi, si le magistrat apporte son expérience personnelle ainsi que ces exigences éthiques et déontologiques, la pratique de l'expertise, notamment psychiatrique et psychologique, implique un rapport au réel amenant sans cesse le professionnel du droit à préférer l'un des champs d'influence distingués dans cet article.

\section{Références :}

[1] Baab, F. L'expertise judiciaire civile en droit allemand. Conférence de consensus sur l'expertise judiciaire civile. Groupe d'analyse des textes. 2007. Available from: URL: http://www.courdecassation.fr/colloques_activites_ formation_4/2007_2254/civile_droit_10910.html

[2] Béliveau P, Pradel J. La justice pénale dans les droits canadien et français : étude comparée d'un système accusatoire et d'un système inquisitoire. Montréal : Yvon Blais ; 2007.

[3] Blumrosen A, Hertz-Béjot B. L'expertise judiciaire civile en droit américain. Conférence de consensus sur l'expertise judiciaire civile. Groupe d'analyse de textes. 2007. Available from: URL : http://www.courdecassation.fr/IMG/File/ pdf_2007/conf_de_consensus/bibliographie/consensus_blumrosen_expertise_dt_americain.pdf

[4] Chauvaud F. Experts et expertise judiciaire en France. Rennes : PUR ; 2003.

[5] Cullaffroz-Jover S. L'expertise en procédure civile espagnole : un pas vers la libéralisation des moyens de preuve ? 2008. Available from : URL : http://m2bde.u-paris10.fr/content/l'expertise-en-procédure-civile-espagnole-un-pas-versla-libéralisation-des-moyens-de-preuve

[6] Dumoulin L. L'expertise judiciaire dans la construction du jugement : de la ressource à la contrainte. Droit et Société $2000 ; 44 / 45: 199-223$.

[7] Encinas de Munagorri R. La recevabilité d'une expertise aux Etats-Unis. Revue internationale de droit comparé $1999 ; 51(3): 621-632$.

[8] Ertaux P, Monsat C. L'expertise psychiatrique au Pénal ou la gestion du crime. Rapport PRC INSERM-CNRS, ${ }^{\circ}$ $132017 ; 1986$.

[9] Faucher P. Quelles sont les attentes du juge de l'application des peines dans l'expertise de pré-libération? In: Audition publique : Expertise psychiatrique pénale. 2007.

[10] Garapon A. (sous la dir.). Incertitude et expertise : l'expertise française sous le regard international. In : Séminaire risques, assurances, responsabilités : le traitement juridique et judiciaire de l'incertitude. 2004-2005

[11] Garapon A, Papadopoulos I. Juger en Amérique et en France. Paris : Odile Jacob ; 2003.

[12] Moulin V. Qui sont les experts psy ? Sciences humaines. 2011-2012; $25: 62-65$.

[13] Nadaud-Castanie ML. Le droit de la preuve devant le juge civil et l'attractivité économique du droit français. (France, Angleterre et Pays de Galles, Etats-Unis). Ministère de la Justice. Service des affaires européennes et internationales ; 2005.

[14] Senon J-L, Lopez G, Cario R. Psychocriminologie et victimologie. Clinique, prise en charge, expertise. Paris : Dunod ; 2008.

[15] Villerbu LM (Dir.) et col. La co-construction expertale. Déconstruction d'une relation paradoxale. Mission de Recherche Droit et Justice ; 2009.

Conflit d'intérêt : aucun. 


\section{RESUME}

Cet article interroge les critères de choix d'un expert judiciaire par les magistrats en charge d'un dossier, à partir d'une étude empirique. Après un détour par l'international afin de spécifier les positions de ces deux acteurs sur la scène judiciaire française, les principaux critères sont décrits. Parmi eux, la célérité, la confiance, la spécialité ou la discipline démontrent que le choix de l'expert s'effectue selon plusieurs dimensions, procédurales bien sûr, mais aussi axiologiques d'une part et expérientielles d'autre part. 